\title{
Gesundes mobiles Arbeiten mit digitalen Assistenzsystemen im technischen Service (ArdiAS)
}

\author{
Rüdiger Mecke, Simon Adler, Daniel Jachmann, Maria Weigel, \\ Steffen Eichholz, Sonja Schmicker, Eric Mewes, Irina Böckelmann (1), \\ Annemarie Minow und Annette Bergmüller
}

\subsection{Einordnung digitaler Assistenzsysteme}

Mobile Endgeräte, wie Smartphones, Tablets und Smartwatches, werden bei Tätigkeiten im industriellen Umfeld zunehmend als digitale Assistenzsysteme eingesetzt. Treiber sind hierbei auch die breite Verwendung im privaten Umfeld sowie die hohe Innovationsrate im Consumer-Markt. Kognitionsunterstützende digitale AS dienen vor allem der anwendungsgerechten, echtzeitnahen Bereitstellung von Informationen, die die Beschäftigten bei Entscheidungen unterstützen oder automatisiert Entscheidungen treffen [1]. Es bestehen wesentliche Unterschiede, je nachdem, ob AS bzw. Bildschirmgeräte ortsgebundenen oder ortsveränderlich verwendet werden (s. Abb. 3.1).

Recherchen zu den rechtlichen Grundlagen der Arbeitsgestaltung für das mobile (ortsveränderliche) Arbeiten mit digitalen AS ergaben, dass es kaum bzw. nicht unmittelbar übertragbare Handlungsempfehlungen gibt. Die aktuelle Arbeitsstättenverordnung (ArbStättV) beschreibt sehr allgemeine Anforderungen an tragbare Bildschirmgeräte für die ortsveränderliche Verwendung an Arbeitsplätzen. Die DGUV Information [2] ent-

R. Mecke $(\bowtie) \cdot$ S. Adler $\cdot$ D. Jachmann

Fraunhofer-Institut für Fabrikbetrieb und -automatisierung IFF, Magdeburg, Deutschland

M. Weigel

Dr. Weigel Anlagenbau GmbH, Magdeburg, Deutschland

S. Eichholz

Terrawatt Planungsgesellschaft mbH, Leipzig, Deutschland

S. Schmicker · E. Mewes

Mensch-Technik-Organisation-Planung - METOP GmbH, Magdeburg, Deutschland

I. Böckelmann · A. Minow · A. Bergmüller

Otto-von-Guericke-Universität Magdeburg, Bereich Arbeitsmedizin, Magdeburg, Deutschland

(C) Der/die Autor(en) 2021

W. Bauer et al. (Hrsg.), Arbeit in der digitalisierten Welt,

https://doi.org/10.1007/978-3-662-62215-5_3 


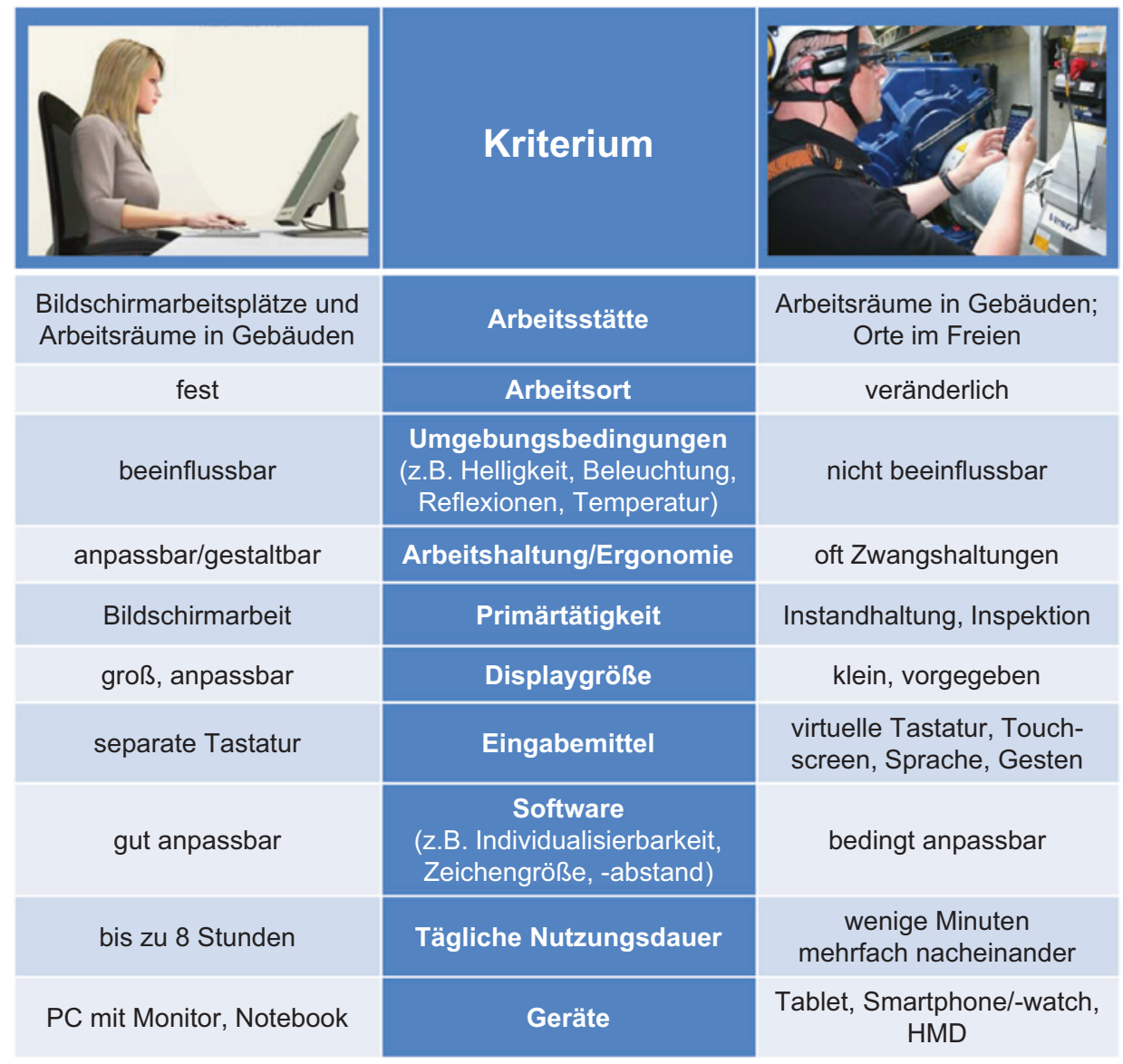

Abb. 3.1 Wesentliche Kriterien und deren Ausprägung bei ortsgebundener und ortsveränderlicher Verwendung von Bildschirmgeräten im Vergleich

hält konkretisierende Gestaltungsempfehlungen technischer Art insbesondere für das Arbeiten mit Notebooks. Aktuell intensiv genutzte mobile Endgeräte, wie z. B. Tablets und Smartphones, werden darin nur kurz behandelt. Endgeräte für den Einsatz an veränderlichen Arbeitsorten, wie Smartwatches und Datenbrillen, sind dort nicht enthalten. Im Vorhaben wurde ein Leitfaden erarbeitet, der auf die nutzerzentrierte ergonomische Gestaltung und schädigungslose Nutzung mobiler Assistenzsysteme im technischen Service Bezug nimmt. 


\subsection{Anwendungsszenarien}

Im Fokus von ArdiAS standen zwei konkrete Anwendungsszenarien (s. Abb. 3.2), anhand derer die menschzentrierte Gestaltung sowie die gesundheitsförderliche und effiziente Verwendung mobiler Assistenzsysteme untersucht wurden.

Beide Szenarien sind gekennzeichnet durch häufig wechselnde Arbeitsorte und kaum beinflussbare Umgebungsbedingungen. Im Vergleich $\mathrm{zu}$ anderen Arbeitssystemen wechselt im mobilen Service die physische Umgebung inklusive potenzieller Gefahrenstellen ständig. Dies verlangt den Mitarbeitern besondere Aufmerksamkeit und Anpassungsfähigkeit $\mathrm{ab}$. An Instandhaltungsarbeitsplätzen halten sich außerhalb der Wartungszyklen nur selten Personen auf. Hieraus folgt, dass bei der Planung solcher Anlagen kaum Wert auf die Ergonomie im Wartungseinsatz gelegt wird. Dies äußert sich z. B. in Zwangspositionen und widrigen Umgebungsbedingungen. Zudem ist an vielen Einsatzorten keine moderne IT-Infrastruktur (mobiles Internet, WIFI) vorhanden.

\subsubsection{Wartung von Industrieanlagen}

Der Unternehmensfokus der Dr. Weigel Anlagenbau GmbH liegt auf der Konzeption, Planung, Realisierung und servicemäßigen Betreuung von Druckluft-, Kühlwasser- und Sonderanlagen. Dazu gehören u. a. die regelmäßige Inspektion und Wartung dieser
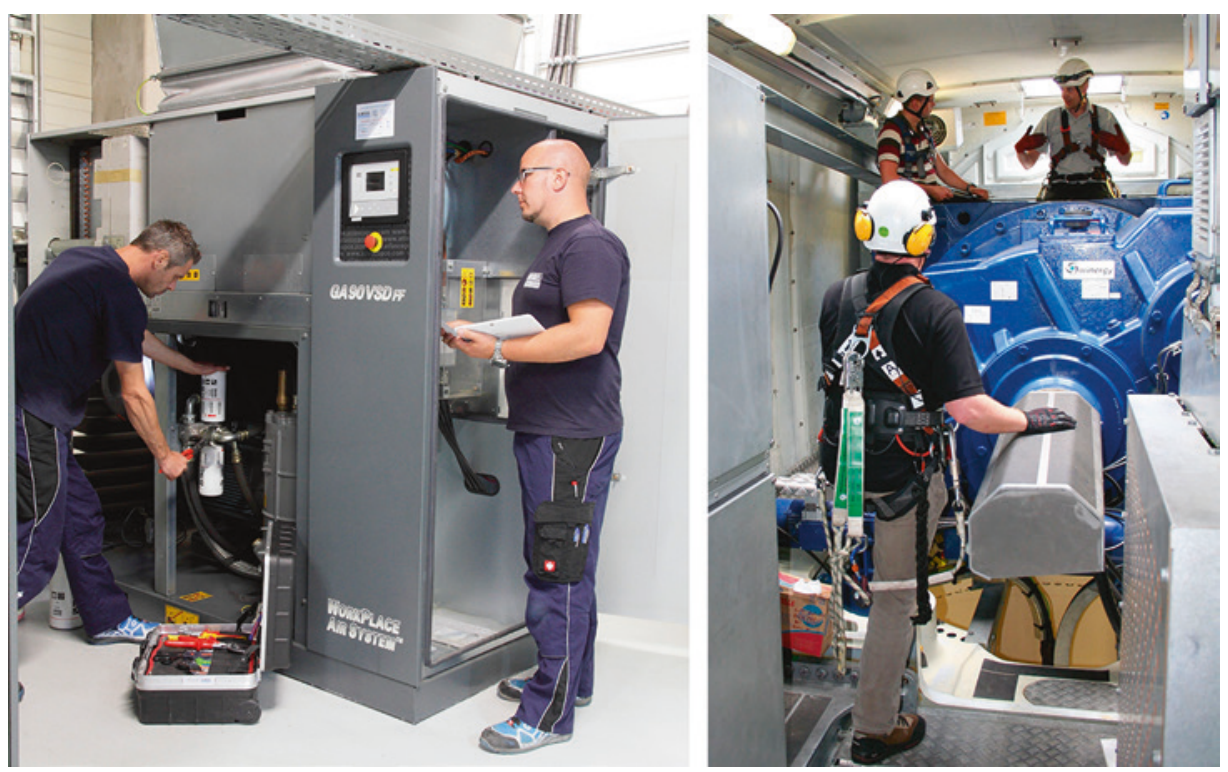

Abb. 3.2 Wartung einer Industrieanlage/Kompressorenstation (links) und Inspektion einer Windenergieanlage (rechts) 
Anlagen (s. Abb. 3.2, links), wofür die Servicekräfte täglich zu den verschiedensten Kunden reisen. Einen typischen Arbeitstag beginnen die Servicemonteure mit dem Abholen der Arbeitsscheine (in Papierform) sowie der notwendigen Ausrüstungen und Ersatzteile in der Firma. Dem Arbeitsschein entnimmt der Monteur den Kunden, den zu kontaktierenden Ansprechpartner vor Ort und die Art der durchzuführenden Arbeit. Die Ersatzteile werden im Regelfall vorkommissioniert vom Anlagenhersteller bezogen. Vor der Abfahrt sind diese von der jeweiligen Servicekraft auf Vollständigkeit zu prüfen sowie ggf. zu vervollständigen. Jeder Monteur führt zudem ein Laptop mit, auf dem er u. a. Datenblätter zu Bauteilen, Schaltpläne und Informationen zu Steuerungen der Anlagen abrufen kann. Beim Kunden angelangt, wird die Anlage zunächst abgeschaltet und Details zur Anlagenhistorie (u. a. Betriebsstunden seit der letzten Wartung, besondere Vorkommnisse) erfasst. Je nach Umfang führen ein oder zwei Monteure anschließend die entsprechenden Wartungsarbeiten durch. Nach der Überprüfung erfolgt ein Testlauf der Anlage in Anwesenheit des Kunden. Die durchgeführten Arbeiten, An-/ Abreisezeitpunkt, wichtige Anlagenwerte und Bemerkungen werden auf dem Arbeitsschein erfasst und vom Kunden quittiert. Wieder auf dem heimischen Firmengelände angelangt, übergibt der Monteur den Arbeitsschein. Die Auftragsdaten werden dann von der Serviceabteilung in das firmeninterne Auftragsverwaltungs- und Organisationssystem übernommen.

Es bestand der Bedarf nach einem digitalen AS, das sowohl die Servicetätigkeit beim Kunden als auch die interne Auftragsverwaltung und Anlagendokumentation der Firma unterstützt. Eine besondere Anforderung war es, die Expertise der Monteure bei der Systemgestaltung einfließen zu lassen.

Während des Montageprozesses kommen die Monteure ständig in Kontakt mit verschiedensten Schmiermitteln. Der Lärmpegel während der Montage unterscheidet sich je nach kundenabhängiger Arbeitsumgebung teilweise stark. Bei Kompressorenstationen liegt meist ein hoher Lärmpegel vor, sodass die Mitarbeiter in der Regel Gehörschutz tragen. Die klimatischen Bedingungen (Temperatur, Luftfeuchtigkeit) in den Werkhallen variieren stark. Dadurch ergaben sich an das digitale Assistenzsystem entsprechende technische Anforderungen, die zusammen mit den benötigten Funktionalitäten zu Projektbeginn spezifiziert wurden.

\subsubsection{Inspektion von Windenergieanlagen}

Die TERRAWATT Planungsgesellschaft $\mathrm{mbH}$ erbringt als Ingenieurdienstleister Inspektionen und Untersuchungen an Windenergieanlagen (WEA) und angeschlossenen elektrotechnischen Einrichtungen. Bei der Inspektion werden WEA im Halbjahresrhythmus auf ihre Funktionstüchtigkeit und mögliche Mängel untersucht (s. Abb. 3.2, rechts).

Vor Beginn der eigentlichen Inspektion sind einige Vorbereitungen notwendig. Zunächst sind die zu untersuchenden Anlagen auszuwählen. Hierbei wird neben der 
Einhaltung des Inspektionszyklus' und dem Vorliegen günstiger Wetterbedingungen (u. a. Wind, Temperatur) auch besonders auf lokale Nähe der Anlagen zueinander geachtet. Zudem wird ein Termin für die Netzabschaltung mit dem Kunden (Betreiber der WEA) abgestimmt. Dann erfolgte eine Überprüfung des Anlagenbetriebs der zurückliegenden Wochen per Ferndiagnose. Ziel ist die Identifikation möglicher Fehler und anderer Auffälligkeiten im laufenden Anlagebetrieb. Zudem werden vergangene Wartungsprotokolle eingesehen, um dann während der Inspektion zu kontrollieren, ob früher identifizierten Mängel beseitigt wurden [3].

Am Tag der Inspektion werden die auszufüllenden Prüfprotokolle (Papier) sowie die nötige Ausrüstung zusammengetragen. Dazu gehören u. a. witterungsgerechte Kleidung, Universalwerkzeuge, Schutzhelm mit Helmlampe und Arbeitshandschuhe. Aufgrund der Arbeitshöhe und verschiedenen Arbeitsstellen mit Sturzgefahr sind die Inspekteure mit einer Fallschutzausrüstung ausgestattet. Diese besteht aus einem Klettergeschirr mit Sicherheitshaken und einem anlagenspezifischen Fallschutzläufer. Weiterhin wird ein Fotoapparat zur Dokumentation von Mängeln benötigt.

Der Ablauf der Inspektion ist gemäß Inspektionsprotokoll in verschiedene Abschnitte der WEA unterteilt. So werden im Außenbereich u. a. Schilder, Zuwegung und Fundament überprüft. Im Turmfuß werden technische Funktionalitäten, wie z. B. die Schaltanlage, das Anlagenlogbuch und der Steuerungsrechner der WEA, untersucht. Andere Abschnitte sind das Aufstiegssystem (Leiter, Aufzug), der Turm, der Azimutbereich zwischen Turm und Maschinenhaus, das Maschinenhaus sowie das Maschinenhausdach. Während der Inspektion, teilen sich beide Inspekteure die Prüfschritte untereinander auf. Hierbei führt einer der Inspekteure das Protokoll, der andere teilt diesem im Nachhinein seine Prüfergebnisse mit. In der Praxis tragen die Inspekteure, auch aufgrund der beengten Bedingungen innerhalb der WEA, ihre Ergebnisse meist nach der Überprüfung eines Abschnittes zusammen [3, 4].

Die meisten Prüfvorgänge bestehen aus einer Sichtprüfung und der Vergabe der Mängelklassifikation (in Ordnung: i.O.; nicht in Ordnung: n.i.O.). Zudem werden Informationen zu auftretenden Mängeln notiert und bei Bedarf Fotos relevanter Prüfstelle aufgenommen. Weiterhin werden Betriebs- und Sensordaten der Anlage ausgelesen und notiert. An bestimmten Stellen erfolgt neben der optischen Prüfung auch eine akustische Prüfung. So werden die Schraubverbindungen im Turm mit einem Klopftest überprüft und auch beim An- und Ablauf des Generators achten die Inspekteure auf Irregularitäten im Klang der Maschinen [3].

Während der Inspektion müssen sich die Inspekteure oftmals in ungünstige Zwangspositionen begeben. Zudem ist aufgrund der Schutzausrüstung die Bewegungsfreiheit teilweise eingeschränkt. Es kommt häufig zum Kontakt mit verschiedenen Schmierstoffen. Die beiden Inspekteure kommunizieren meist verbal miteinander. Bei einigen Arbeitsschritten agieren die Mitarbeiter außerhalb des beiderseitigen Sichtbereichs. Die Umgebungsbedingungen in den Windenergieanlagen variieren stark. So bestehen z. B. Lichtverhältnisse von absoluter Dunkelheit bis Tageslicht mit direkter Sonnenein- 
strahlung. Temperaturen liegen im Bereich von $-10{ }^{\circ} \mathrm{C}$ bis $+40{ }^{\circ} \mathrm{C}$. Es kann nicht davon ausgegangen werden, dass bei allen Anlagen eine mobile Internetverbindung besteht. Im Inneren der WEA treten zudem elektromagnetische Abschirmungen sowie Interferenzen auf [3].

Ein digitales Assistenzsystem soll insbesondere die Dokumentation und Qualitätssicherung der Inspektion sowie die Kollaboration der beiden Prüfer unterstützen. Insbesondere die direkte Integration der Fotoerfassung hat Potenzial, den Aufwand bei der Dokumentationserstellung wesentlich zu reduzieren.

\subsection{Entwicklung und Einsatz mobiler Assistenzsysteme}

\subsubsection{Partizipative interdisziplinäre Zusammenarbeit}

Bei der nutzerzentrierten Entwicklung von Assistenztechnologien ist es essentiell, die Anwender sowie alle involvierten Fachdisziplinen von Beginn an einzubeziehen. Die Zusammenarbeit von Technologieentwicklern, Arbeitswissenschaftlern und Arbeitsmedizinern birgt hierbei im Sinne einer ganzheitlichen Betrachtung der Ebenen MenschTechnik-Organisation große Potentiale und Herausforderungen, die im Rahmen des Verbundprojektes ArdiAS adressiert wurden (s. Abb. 3.3).

Im Anwendungsfokus stehen zwei konkrete industrielle Praxisszenarien zur Wartung und Inspektion von technischen Anlagen (s. Abschn. 3.4). Für beide Anwendungen erfolgte zunächst die Analyse der Arbeitsprozesse und Anforderungen [3] sowie auf dieser Basis die Spezifikation des Assistenzsystems. Dazu haben die Forschungspartner aus dem Bereich der Arbeitswissenschaft vor Ort bei den involvierten Unternehmen Prozessanalysen und Befragungen durchgeführt. Dabei wurden sowohl die hohen Anforderungen an die Qualität der Wartungs- und Inspektionstätigkeiten als auch der Bedarf nach digitalen Unterstützungssystemen deutlich. Es zeigte sich, dass partizipative Ansätze unter Einbeziehung der Anwender, wie beispielsweise moderierte Workshops, sehr hilfreich und zielführend sind. Parallel dazu wurden von der IT-Entwicklung (IFF) Recherchen zu aktuellen mobilen Endgeräten (Smartphones, Tablets, Datenbrillen) durchgeführt, um daraus geeignete Geräte für die jeweilige Anwendung auszuwählen. Auch hierbei war es sehr zielführend, den Anwendern neueste Trends aus der Geräteentwicklung vorzustellen und frühzeitig die Eignung für den Praxiseinsatz abzuschätzen.

Eine weitere zentrale Fragestellung im arbeitsmedizinischem Kontext bestand darin, objektive physiologische Indikatoren für die Arbeitsbeanspruchung der Beschäftigten bei der kombinativen Arbeit zu ermitteln. Es fanden hierfür Laborstudien [5-7] statt, um die Kenngrößen der kognitiven Beanspruchung zu ermitteln. Dabei wurden für die Erfassung der objektiven Beanspruchung das Elektrokardiogramm (EKG) und daraus abgeleitete Kenngrößen (Herzratenvariabilität, HRV) sowie das Elektroenzephalogramm (EEG) genutzt. Die subjektive Beanspruchung wurde anhand standardisierter Verfahren 


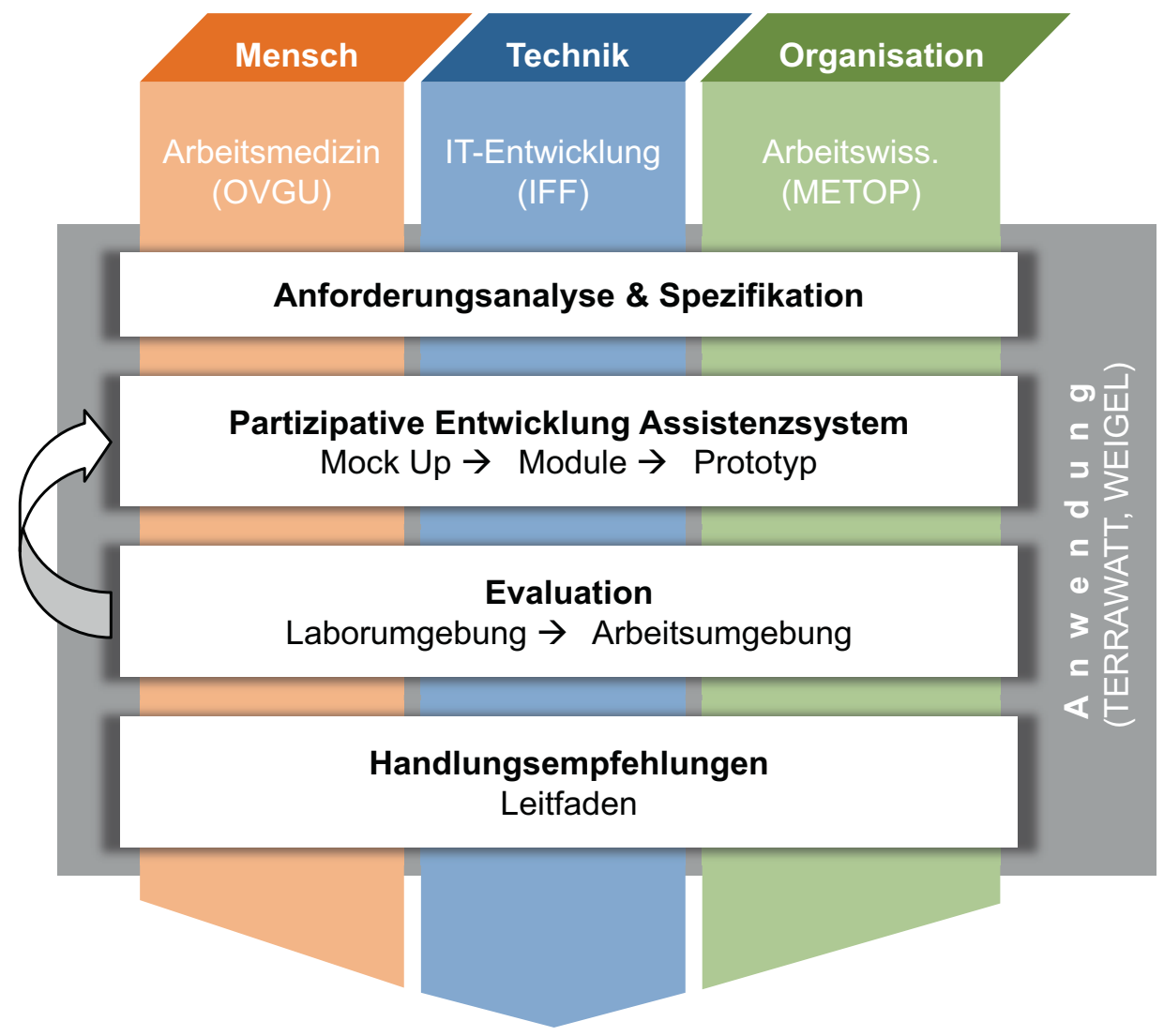

Abb. 3.3 Interdisziplinarität bei der partizipativen Entwicklung und Evaluation digitaler Assistenzsysteme

abgefragt. In einer weiteren Laborstudie [8, 9] erfolgte die Analyse der Auswirkungen unterschiedlicher Bildschirmtypografien auf die visuelle Beanspruchung, die kognitive Leistungsfähigkeit und die subjektive Beanspruchung. Die Erkenntnisse aus diesen Studien fanden bei den arbeitswissenschaftlichen Untersuchungen sowie der Evaluation der Prototypen der mobilen AS unter Labor- und realen Arbeitsbedingungen Berücksichtigung.

Die technische Entwicklung des Assistenzsystems beinhaltete insbesondere die softwareseitige Implementierung der spezifizierten Funktionalitäten sowohl für die mobilen Endgeräte als auch für die dahinterliegende Serverinfrastruktur. Die Implementierungen erfolgten hierbei modulweise jeweils ausgehend von entsprechenden Mock Ups (s. Abb. 3.3). Bei diesen handelt es ich um Demonstrationsmodelle, mit denen wesentliche Programm- und Bedienfunktionalitäten den involvierten Partnern auf einfache 
Art präsentiert werden. Potentielle Anwender können somit frühzeitig in die Systementwicklung einbezogen werden und konkrete Wünsche und Ideen aus Praxissicht einbringen. Der Prototyp des Assistenzsystems wurde aus einzelnen anwendungsspezifischen Modulen erstellt und enthielt eine exemplarische Anbindung an die bei den Anwendungspartnern bestehende IT-Infrastruktur. Nur so kann das System später auch anhand von betrieblichen Praxisszenarien evaluiert werden. Während der technischen Entwicklung werden in der Regel mehrere Iterationsschleifen sowohl auf Modul- als auch Prototypenebene durchlaufen.

Das Ziel der Evaluation (s. Abb. 3.3) ist es, die Prototypen in konkreten Anwendungsumgebungen zu testen. Die Untersuchungen fanden dabei zunächst in einer Laborumgebung statt $[4,10]$, in der vereinfachte Inspektionsaufgaben von anwendungsfernen Probanden durchgeführt und analysiert wurden (Abb. 3.4, links). Dies dient zur Einschätzung der prinzipiellen Eignung und liefert Feedback für die Systemoptimierung (s. Abb. 3.3, Feedbackschleife zur Systementwicklung). Dann wurden Serviceexperten aus den Anwendungsunternehmen einbezogen, die den neuen Technologien besonders aufgeschlossen gegenüberstanden. In dieser Phase war die Unterstützung technologieaffiner Promotoren besonders wichtig. Mit den optimierten Prototypen erfolgten dann Tests in der realen Arbeitsumgebung (s. Abb. 3.4, rechts) durch die Serviceexperten der Unternehmen.

\subsubsection{Struktur und Funktionalitäten des Assistenzsystems}

Die Systemarchitektur des Assistenzsystems besteht aus verschiedenen Modulen. Ausgewählte Kernmodule und deren Zusammenwirken sind im nachfolgenden Schema veranschaulicht (s. Abb. 3.5). Auf einem stationären Server läuft ein webbasiertes
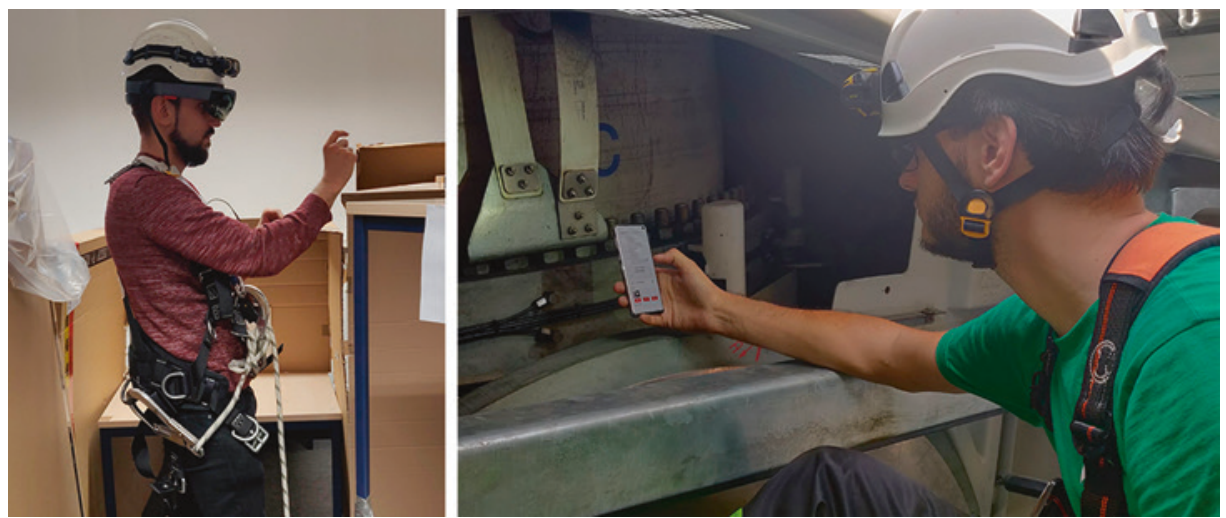

Abb. 3.4 Evaluation von Protoypen der mobilen AS in Laborumgebung (links) und realer Arbeitsumgebung (rechts) 


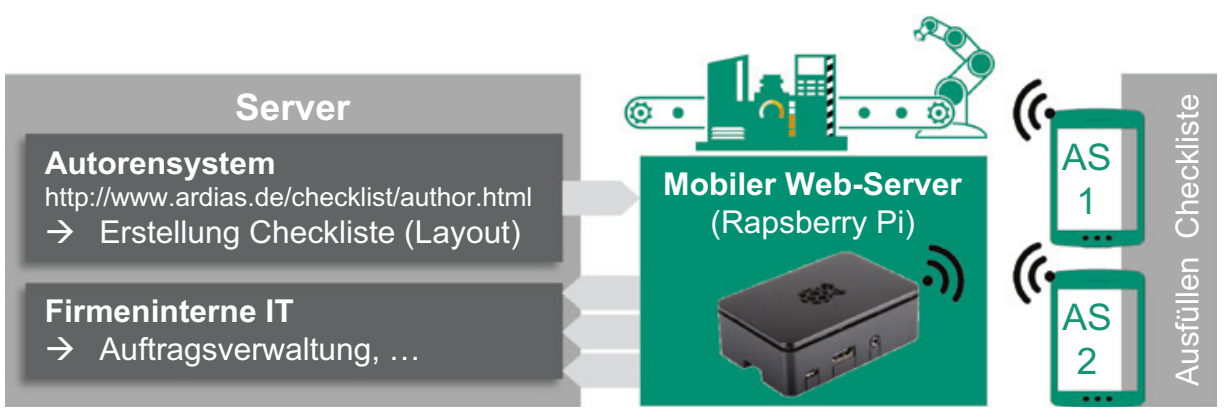

Abb. 3.5 Systemarchitektur des Assistenzsystems (vereinfachte Darstellung)

Autorensystem, mit dem die Anwender selbstständig kunden- und anlagenspezifische Checklisten erstellen können. Diese enthalten leere Prüfprotokolle sowie weitere Informationen zur durchzuführenden Tätigkeit und werden auf einen mobilen WebServer (Raspberry Pi) geladen. Dieser Server wird in der Arbeitsumgebung mitgeführt und stellt per WLAN eine Anbindung für mobile Endgeräte (AS1, AS2) zur Verfügung. Beim zeitgleichen Zugriff von mehreren Endgeräten werden bei Web-Anwendungen üblicherweise die einzelnen Verbindungen isoliert behandelt. Im vorliegenden System wurde diese Isolation aufgehoben, damit mehrere Nutzer kollaborativ an einem gemeinsamen Prüfprotokoll arbeiten können. Es wird sichergestellt, dass die Eingaben eines Nutzers innerhalb weniger Sekunden an alle weiteren Endgeräte weitergeleitet und dort visualisiert werden. Bei Abbruch der WLAN-Verbindung zum mobilen Web-Server kann das Prüfprotokoll auch ohne Netzwerkverbindung auf den Endgeräten bearbeitet werden. Die erfolgten Eingaben werden dann lokal auf den Endgeräten zwischengespeichert und nach erneutem Verbindungsaufbau synchronisiert. Das Protokoll wird nach Abschluss der Tätigkeit in einer Datenbank auf dem mobilen Web-Server gespeichert und kann von dort aus in den gängigen Dateiformaten in die firmeninterne IT-Infrastruktur übertragen werden.

Im Kontext der Datensicherheit ist hervorzuheben, dass für die Nutzung des Systems keine Verbindung zum Internet erforderlich ist, sondern der Raspberry Pi per Kabel ausgelesen werden kann. Die softwaretechnischen Umsetzungen basieren auf etablierten Web-Technologien (Angular, Nodejs). Damit liegt eine hohe Einsatzflexibilität bezüglich verschiedener mobiler Endgeräte (Betriebssysteme Android, iOS, Windows) vor.

Beim Abarbeiten der Checkliste auf dem mobilen AS (s. Abb. 3.6) erfolgt die Navigation über ein Inhaltsverzeichnis der einzelnen Prüfschritte, das über eine Schaltfläche ein- und ausgeblendet wird (a). Man kann einzelne Schritte der Checkliste auch über Navigationspfeile auswählen (b). Zu jedem Prüfschritt können das Prüfergebnis als Mängelklassifikation eingegeben (c) sowie Fotos angefügt (d) werden. In den Fotos lassen sich Markierungen einzeichnen. Es ist möglich, zusätzliche Informationen als Kommentare in Freitextform (e) und gegebenenfalls als Schlüssel-Wert-Paare zu 
erfassen. In den Prüfschritten können optional Arbeitsinstruktionen und beschreibende Bilder angezeigt werden, die zuvor mit dem Autorensystem erstellt wurden. Nach Abarbeitung aller Schritte wird die Checkliste vom Mitarbeiter als abgearbeitet markiert und steht dann als Dokument zur Verfügung, das z. B. in ein PDM-System transferiert werden kann.

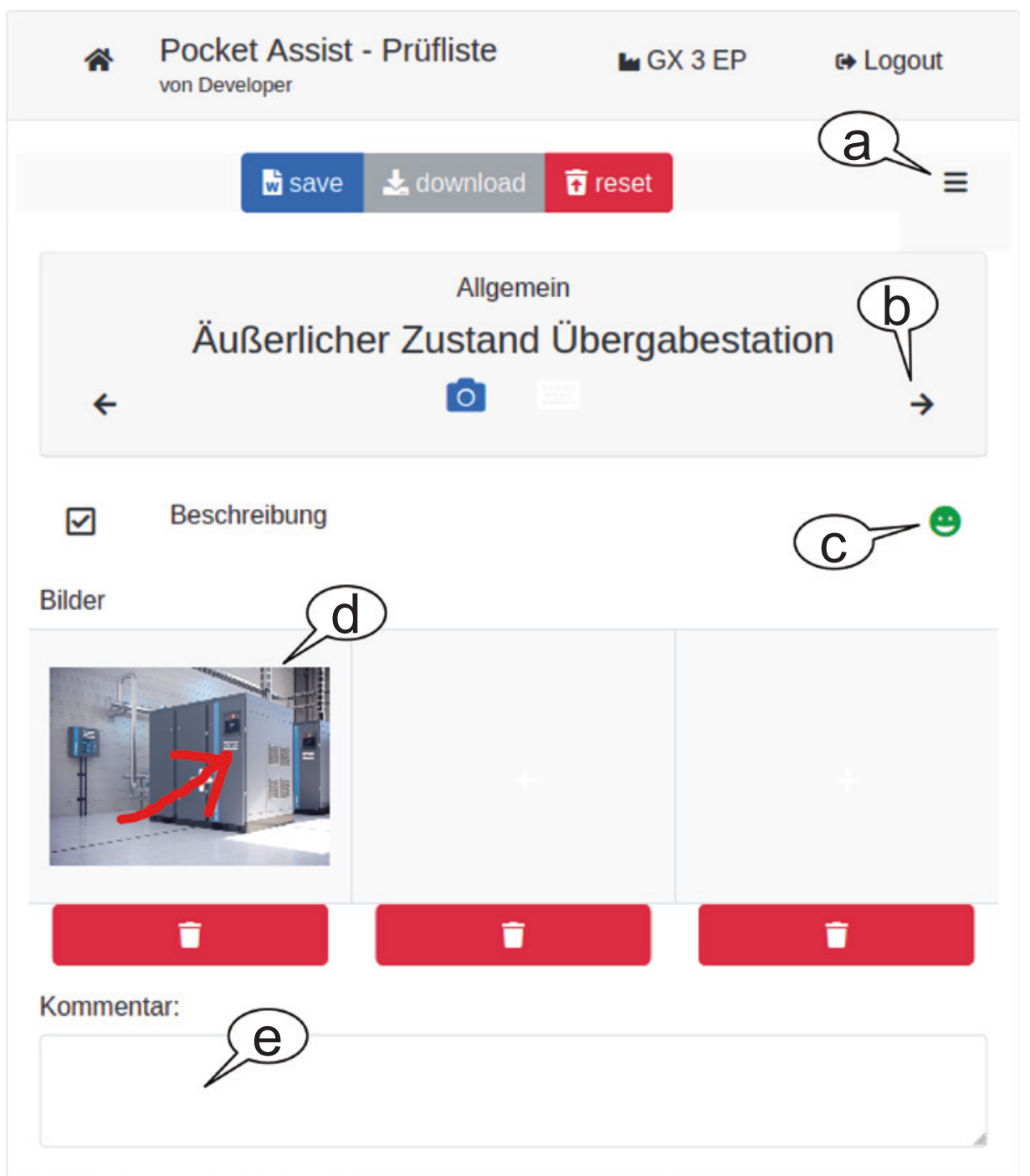

Abb. 3.6 Funktionalitäten des Assistenzsystems am Beispiel eines Prüfschrittes in einer Checkliste 
Neben diesen Kernfunktionalitäten des Systems wurden weitere Unterstützungsfunktionen realisiert, die für die praxisnahe Evaluation und perspektivische Integration in die Workflows der Anwendungspartner erforderlich sind. Hierzu gehören die Verwaltung von Auftragsdaten, Maschinen- und Anlagentypen, Prüflistenvorlagen und Benutzern. Außerdem wurden Backup- und Aktualisierungsfunktionen umgesetzt. Die Navigation in der Anwendung wurde für mobile Geräte sowie Desktop-PCs optimiert.

\subsubsection{Arbeitswissenschaftliche Untersuchungen und Evaluierung}

Je nach Arbeitsinhalt und -umgebung bestehen verschiedene Anforderungen, welche sich sowohl auf die Auswahl des Endgeräts als auch die Interaktionsgestaltung zwischen Mensch und Maschine auswirken. Zur Systematisierung wurde eine Expertenbefragung mit internationaler Beteiligung zu den Vor- und Nachteilen der ausgewählten Endgeräte durchgeführt [11, 12]. Die Ergebnisse der Befragung sind hier in vereinfachter und gekürzter Form zusammengefasst dargestellt (Tab. 3.1).

Um eine menschgerechte Beanspruchung der Beschäftigten zu ermöglichen, sollten dargestellte Informationen im Assistenzsystem sowohl an die jeweiligen Arbeitsinhalte, als auch an den individuellen Kenntnisstand des Nutzers angepasst sein. Auf diese Weise lässt sich sicherstellen, dass die Arbeitspersonen zur richtigen Zeit mit den richtigen Informationen versorgt werden. Gerade bei der Ersteinführung sind optimierte Umfänge und Darstellungen der Informationen für die Akzeptanz der Assistenzsysteme von besonderer Bedeutung. Besonders bei Mitarbeitern mit wenig Berufserfahrung sind während der fortlaufenden Ausübung der Tätigkeit die Bildung neuer Kompetenzen zu erwarten. Dadurch können sich auch die jeweiligen Anforderungen an das Assistenzsystem verändern. Beispielsweise kann eine genaue Aufschlüsselung der Arbeitsschritte zu Beginn der Tätigkeit Unsicherheiten beim Beschäftigten entgegenwirken. Nachdem dieser mit dem Arbeitsablauf vertraut ist, sollte sich der Unterstützungsgrad reduzieren lassen. Generell ist bei der Konzeption darauf zu achten, dass durch das System die Kompetenzbildung gefördert und die Gefahr des Kompetenzverlusts minimiert werden.

Digitale Assistenzsysteme ermöglichen vollkommen neue Formen der Arbeitsorganisation, welche den Mitarbeitern entsprechende Kompetenzen abverlangen. Zu bedenken sind hierbei auch eine mögliche Überforderung durch zusätzliche oder veränderte Arbeitsinhalte und Arbeitsunterbrechungen durch ständige Erreichbarkeit.

\subsubsection{Arbeitsmedizinische Beanspruchungsuntersuchungen}

Die beanspruchungsoptimale Gestaltung digitaler Assistenzsysteme erfordert möglichst objektive Indikatoren zur Beurteilung der Arbeitsbeanspruchung der Beschäftigten. Beim technischen Service liegen vor allem kombinatorischen Tätigkeiten mit visuellen und kognitiven Anforderungen vor. Durch die Partner aus dem Bereich der Arbeits- 
Tab. 3.1 Expertenbewertung zur Eignung von Endgeräten in Abhängigkeit ausgewählter Kriterien; reduzierte Darstellung nach [11] (Legende: ++ sehr gut, + gut, o kein Vorteil, schlecht, - - sehr schlecht); Quelle: METOP GmbH

\begin{tabular}{|c|c|c|c|c|c|}
\hline Kriterium & Smartphones & Tablets & Smartwatches & $\begin{array}{l}\text { Binocular Smart } \\
\text { Glasses }\end{array}$ & $\begin{array}{l}\text { Monocular } \\
\text { Smart Glasses }\end{array}$ \\
\hline $\begin{array}{l}\text { Transportauf- } \\
\text { wand }\end{array}$ & + & - & ++ & - & - \\
\hline Kamerafunktion & ++ & + & -- & - & - \\
\hline $\begin{array}{l}\text { Vibrations- } \\
\text { funktion }\end{array}$ & + & - & ++ & -- & -- \\
\hline Tonausgabe & + & - & -- & + & + \\
\hline $\begin{array}{l}\text { Einschränkungs- } \\
\text { freiheit der } \\
\text { Umgebungs- } \\
\text { wahrnehmung } \\
\text { des Nutzers }\end{array}$ & + & + & ++ & o & o \\
\hline $\begin{array}{l}\text { Bedienfreund- } \\
\text { lichkeit }\end{array}$ & ++ & ++ & + & - & - \\
\hline $\begin{array}{l}\text { Freiheit von } \\
\text { Mehrbelastung } \\
\text { durch Nutzung }\end{array}$ & + & + & ++ & - & - \\
\hline $\begin{array}{l}\text { Nutzung mit } \\
\text { Handschuhen }\end{array}$ & - & - & - & + & + \\
\hline $\begin{array}{l}\text { Nutzung mit } \\
\text { Schutzhelm }\end{array}$ & + & + & + & - & - \\
\hline $\begin{array}{l}\text { Ausgabe } \\
\text { Wörter/Ziffern/ } \\
\text { Piktogramme }\end{array}$ & + & + & + & + & + \\
\hline $\begin{array}{l}\text { Ausgabe Sätze/ } \\
\text { Bilder/Clips }\end{array}$ & + & + & - & + & + \\
\hline $\begin{array}{l}\text { Ausgabe } \\
\text { Fließtext/ } \\
\text { komplexe } \\
\text { Bilder/Videos }\end{array}$ & + & ++ & - & + & + \\
\hline $\begin{array}{l}\text { Darstellung von } \\
\text { Augmented } \\
\text { Reality }\end{array}$ & + & + & -- & ++ & + \\
\hline $\begin{array}{l}\text { Nutzung mit } \\
\text { Schutzhelm }\end{array}$ & + & + & + & - & - \\
\hline
\end{tabular}


medizin wurde die Herzfrequenzvariabilität (HRV), die aus dem Elektrokardiogramm (EKG) abgeleitet wird, als Beanspruchungsindikator untersucht. Aus den RR-Intervallen (Abstand zwischen zwei R-Zacken) des EKG kann neben der Herzschlagfrequenz auch deren Variabilität berechnet werden, die eine Quantifizierung des individuellen Beanspruchungszustandes im Verlauf des Arbeitsprozesses erlaubt. Mit Hilfe verschiedener Parameter der HRV ist es möglich, das Zusammenspiel von Sympathikus und Parasympathikus bei der Regulation und Steuerung des Herz-Kreislauf-Systems, u. a. bei unterschiedlichen Belastungssituationen im Arbeitsprozess, differenziert zu beschreiben (s. Abb. 3.7). Im oberen Teil der Abbildung findet sich der Herzschlag pro Minute (grün: Mittelwert, rot/blau: obere/untere Hüllkurve). Im unteren Teil sind HRVSpektren für 2.5-minütige Zeitintervalle dargestellt. Die Farbe gibt einen Hinweis zur Intensität der Variabilität; blau und rot als intensive Farben bedeuten eine hohe Variabilität bei dem entsprechenden Frequenzspektrum. Die Aktivität des Sympathikus, die in den Belastungsphasen größer ist, findet man im Bereich bis $0,15 \mathrm{~Hz}$, also nahe der Zeitachse. Sympathikus und Parasympathikus sind Teil des vegetativen Nervensystems. Diese Nerven werden funktionell als Gegenspieler betrachtet. Die HRV ist ein Parameter der allgemeinen Aktivierung und der sympatho-vagalen Balance des Organismus‘. Über die Ausprägung des Niveaus der Regulationsmechanismen können die funktionalen Reserven des Herz-Kreislauf-Systems und die Anpassungsmöglichkeiten des Gesamtorganismus beurteilt werden. Hierbei gilt vereinfacht: je gleichmäßiger/ungleichmäßiger die Herzschlagfrequenz ist, desto höher/geringer ist die Beanspruchung.

Als weitere Beanspruchungsindikatoren wurden die Spektralleistungsparameter aus dem Elektroenzephalogramm (EEG) eingesetzt, die Aufschlüsse über die Gehirnaktivität unter kognitiver Belastung geben. Die Zusammenhänge zwischen diesen objektiven physiologischen Beanspruchungsindikatoren sowie die Assoziationen mit der subjektiven Beanspruchung und objektiven Leistung bei kognitiven Aufgaben wurden analysiert. Sowohl bei den Auslenkungen der HRV-Parameter als auch bei den Änderungen in den spektralen EEG-Parametern wurden Anpassungsreaktionen an die standardisierten psychometrischen Tests beobachtet. Hinsichtlich der Beanspruchungskorrelate auf der Herz-Gehirn-Achse sind einige spezifische HRV-Parameter (LF nu, HF nu, pNN50) geeignet, unter bestimmten Kriterien Rückschlüsse auf die per EEGermittelte mentale Beanspruchung zuzulassen.

Aus den Ergebnissen dieser Untersuchungen werden folgende Empfehlungen für die Arbeitsgestaltung gegeben, welche zu einer Optimierung der Belastung Arbeitender führen und die Arbeitsbedingungen verbessern:

Eine gute Einführung der Nutzer in die Anwendung digitaler AS ist aus physiologischer Sicht nötig. Die Untersuchungen zeigten, dass bei der erstmaligen Nutzung von neuen Assistenzsystemen die Beanspruchung zu Beginn erhöht ist. Dieser Effekt verschwindet jedoch, wenn die Personen die Systeme wiederholt nutzen (sog. Phänomen der Habituation). Das repräsentiert zudem auch die schnelle Anpassungsfähigkeit an diese neuen Arbeitsmittel. Eine wesentliche Limitierung bisheriger Studienergebnisse, auch unserer, ist der kurzfristige Einsatz digitaler AS. Um valide Aussagen über die lang- 


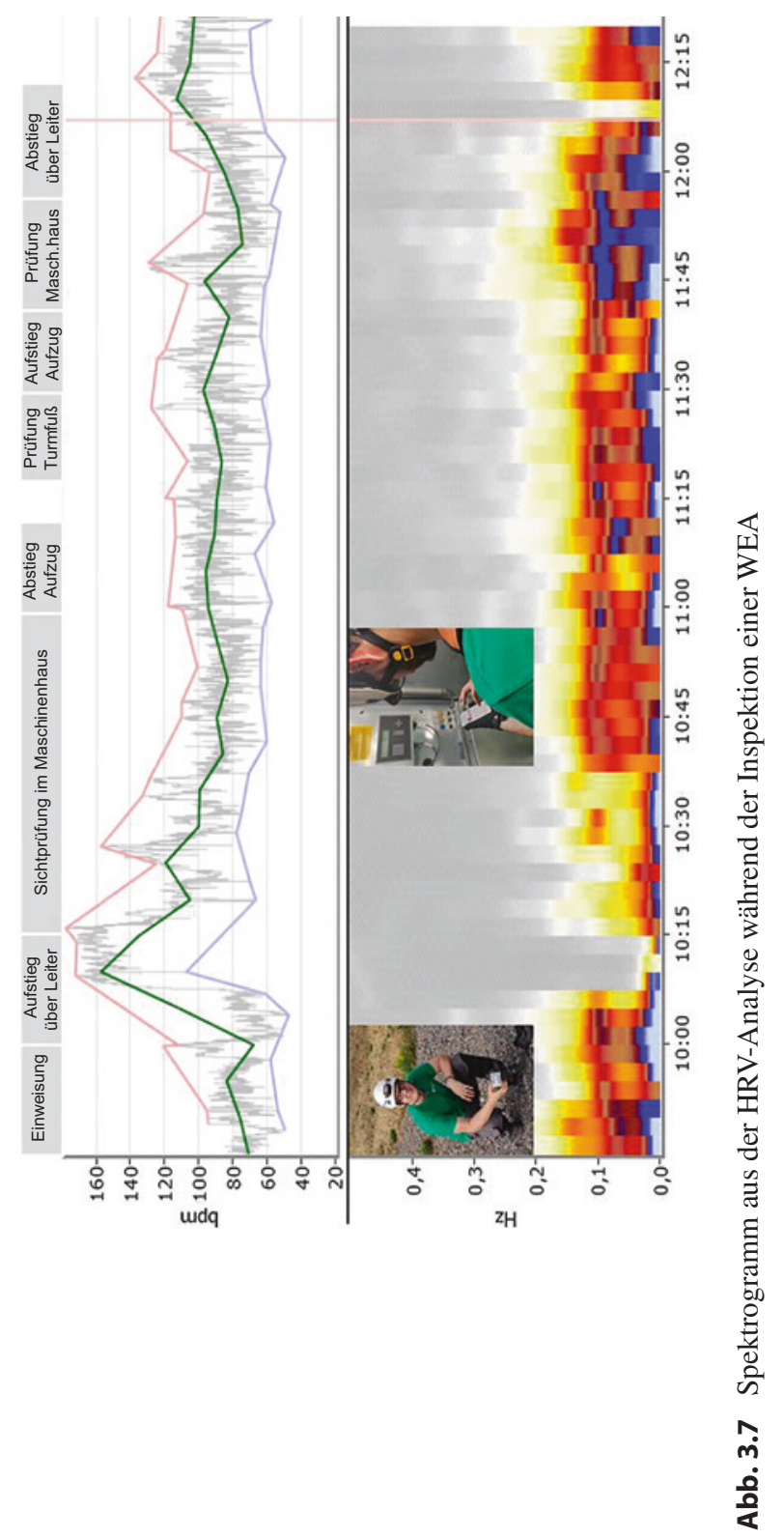


fristigen Beanspruchungsfolgen treffen zu können, sind weitere Untersuchungen nötig, die eine längere Einsatzdauer (z. B. ein gesamter Arbeitstag) und einen längeren Zeitraum (z. B. mehrere Wochen) umfassen. Auswirkungen digitaler AS auf den Nutzer müssen zudem stets mehrdimensional beurteilt werden. Nicht nur die physiologischen Beanspruchungen und die objektive kognitive Leistungsfähigkeit, sondern auch das subjektive Beanspruchungsempfinden, die Nutzerakzeptanz und Aspekte der Gebrauchstauglichkeit müssen Eingang in die gesundheitliche Beurteilung finden.

\subsection{Hinweis auf Transfermaterialien}

Im Rahmen des Vorhabens wurde ein Leitfaden zur nutzerzentrierten Entwicklung und Nutzung digitaler Assistenzsysteme im technischen Service erarbeitet [13]. Ziel dieses Leitfadens ist die Bereitstellung von Informationen und Hinweisen, um einen beanspruchungsoptimalen Einsatz digitaler AS zu gewährleisten. Im Fokus steht dabei die Verwendung solcher Systeme im technischen Service, der besonders von häufig wechselnden Einsatzorten und flexiblen Arbeitsbedingungen geprägt ist. Damit sollen die Chancen der Digitalisierung besser genutzt und Risiken verringert werden. Neben den potenziellen Anwendern und Arbeitgebern adressiert der Leitfaden insbesondere auch Entwickler von mobilen Endgeräten und Applikationen in Hinblick auf Hard- und Software-Ergonomie.

\subsection{Fazit und Ausblick}

Im Verbundprojekt ArdiAS wurden digitale Assistenzsysteme unter Berücksichtigung der Erkenntnisse der involvierten Fachdisziplinen IT-Entwicklung, Arbeitswissenschaft und Arbeitsmedizin prototypisch implementiert und untersucht. Es erfolge die Integration dieser Demonstratoren in zwei konkrete Anwendungsszenarien im technischen Service sowie deren Evaluation. Hinsichtlich Nutzbarkeit der Systeme und Belastungssituation der Beschäftigten sowie bezüglich wirtschaftlicher Aspekte (z. B. Qualitäts- und Zeitgewinn) wurden positive Effekte nachgewiesen.

Die partizipative Entwicklung und Einführung der AS hat sich als hilfreiches Mittel für die menschgerechte Entwicklung herausgestellt. Dabei ist eine möglichst frühe Einbindung der Mitarbeiter förderlich für die Akzeptanz. In Zukunft sind besonders Langzeitstudien mit dauerhafter Verwendung der Systeme und ihre Einwirkung auf die Arbeitswelten der Zukunft interessant.

Die Ergebnisse der arbeitsmedizinischen Untersuchungen legen nahe, dass es zumindest im kurzfristigen Einsatz und nach einer kurzen Adaptionszeit - keine erhöhte objektiv physiologische Beanspruchung bei der Tätigkeit mit digitalen AS im Vergleich zu herkömmlichen Arbeitsweisen gibt. Eine gute Einführung in neue AS ist essenziell für einen gesundheitsgerechten Einsatz digitaler AS. 
Es ist perspektivisch zu untersuchen, inwieweit die Ergebnisse auch auf andere Anwendungsbereiche und Vorgehensmodelle übertragbar sind. Das partizipative Vorgehen bei der Technologieentwicklung birgt sowohl Herausforderungen als auch hohe Innovationspotenziale, die zukünftig weiterbefördert werden müssen.

Zum Projektende liegt ein Praxisleitfaden zur nutzerzentrierten Entwicklung und Nutzung digitaler Assistenzsysteme im technischen Service vor.

\section{Projektpartner und Aufgaben}

- Fraunhofer-Institut für Fabrikbetrieb und -automatisierung IFF Nutzerzentrierte Entwicklung mobiler Assistenzsysteme für technische Dienstleistungen

- Otto-von-Guericke-Universität Magdeburg, Bereich Arbeitsmedizin Arbeitsmedizinische und psychophysiologische Untersuchungen nutzerbezogener Aspekte

- Mensch-Technik-Organisation-Planung METOP GmbH Arbeitswissenschaftliche Untersuchungen und partizipative Gestaltungskonzepte

- Dr. Weigel Anlagenbau GmbH

Anwendung digitaler Assistenzmethoden bei der Instandhaltung von Industrieanlagen

- Terrawatt Planungsgesellschaft mbH

Anwendung digitaler Assistenzmethoden bei der Inspektion von Windenergieanlagen

\section{Literatur}

1. Apt W, Schubert M, Wischmann S (2018) Digitale Assistenzsysteme - Perspektiven und Herausforderungen für den Einsatz in Industrie und Dienstleistungen. Institut für Innovation und Technik (iit) in der VDI/VDE Innovation+Technik GmbH, Berlin

2. DGUV (2012) Belastungen und Gefährdungen mobiler IKT-gestützter Arbeit im Außendienst moderner Servicetechnik, Handlungshilfe für die betriebliche Praxis - Gestaltung der Arbeit. https://www.uv-bund-bahn.de/fileadmin/Dokumente/Mediathek/211-036.pdf. Zugegriffen: 27. Febr 2020

3. Mewes E, Schmicker S, Waßmann S, Mecke R, Böckelmann I (2018) Entwicklung und Durchführung einer Anforderungsanalyse zur Identifikation von nutzergunterstützenden Anwendungspotenzialen digitaler Assistenzsysteme in mobilen Servicetätigkeiten. In Dokumentation des 64. Arbeitswissenschaftlichen Kongresses, Beitrag B.1.3; Dortmund

4. Mewes E, Waßmann S, Adler S, Minow A, Schmicker S (2019) Entwicklung eines Laboraufbaus zur Erprobung eines digitalen Assistenzsystems für den Einsatz in der mobilen Instandhaltung; In: Arbeit interdisziplinär - Dokumentation des 65. Arbeitswissenschaftlichen Kongresses, Beitrag D.1.5, Frankfurt 
5. Dorn A, Minow A, Darius S, Böckelmann I (2019) Auswirkungen von Aufmerksamkeitstests unterschiedlicher kognitiver Anforderungen auf die Auslenkung der HRV-Parameter . Zentralblatt für Arbeitsmedizin Arbeitsschutz und Ergonomie 70:99-108. https://doi.org/10.1007/ s40664-019-00374-6

6. Schapkin S, Raggatz J, Hillmert M, Böckelmann I (2020) EEG correlates of cognitive load in a multiple choice reaction task. Acta Neurobiol Exp 80:76-89

7. Hillmert M, Bergmüller A, Minow A, Raggatz J, Böckelmann I (2020) Psychophysiologische Beanspruchungskorrelate während kognitiver Belastung: Eine Laborstudie mittels EEG und EKG. Zentralblatt für xArbeitsmedizin, Arbeitsschutz und Ergonomie 70:149-163. https://doi. org/10.1007/s40664-020-00384-9

8. Bergmüller A, Minow A, Adler S, Böckelmann I (2020) Eine Laborstudie zur subjektiven und objektiven visuellen Beanspruchung bei verschieden dargestellten Aufmerksamkeitstests am Smartphone. Abstract 60. Jahrestagung der Deutschen Gesellschaft für Arbeitsmedizin und Umweltmedizin e.V. (DGAUM), 11. - 14. März 2020 in München

9. Minow A, Bergmüller A, Adler S, Böckelmann I (2020) Auswirkungen der Bildschirmtypografie eines Smartphones auf die subjektive Beanspruchung und Leistung bei mobiler Arbeit. Abstract 60. Jahrestagung der Deutschen Gesellschaft für Arbeitsmedizin und Umweltmedizin e.V. (DGAUM), 11. - 14. März 2020 in München

10. Mewes E, Waßmann S, Minow A, Adler S, Schmicker S (2019) Laborversuch zur Validierung der Nutzerfreundlichkeit eines digitalen Assistenzsystems für den Einsatz in der mobilen Instandhaltung

11. Tagungsband 14. Magdeburger Maschinenbau-Tage, Magdeburg, S 320-329. https://opendata. uni-halle.de//handle/1981185920/13829. Zugegriffen: 27. Febr 2020

12. Mewes E, Schwarz F, Wassmann S, Adler S, Schmicker S (2020). Methodik zur Unterstützung der Hardwareauswahl digitaler Assistenzsysteme für mobile, industrielle Servicetätigkeiten. In Tagungsband der 22. IFF Wissenschaftstage. Fraunhofer Verlag, Stuttgart

13. Mewes E, Bergmüller A, Minow A, Waßmann S, Weigel M, Eichholz S, Adler S, Böckelmann I, Schmicker S, Mecke R (2020) Digitale Assistenzsysteme zur mobilen Verwendung im technischen Service in der Instandhaltung - Ein Leitfaden für die Gestaltung und Nutzung. Otto von Guericke University Library, Magdeburg. http://dx.doi.org/10.25673/32943 
Open Access Dieses Kapitel wird unter der Creative Commons Namensnennung 4.0 International Lizenz (http://creativecommons.org/licenses/by/4.0/deed.de) veröffentlicht, welche die Nutzung, Vervielfältigung, Bearbeitung, Verbreitung und Wiedergabe in jeglichem Medium und Format erlaubt, sofern Sie den/die ursprünglichen Autor(en) und die Quelle ordnungsgemäß nennen, einen Link zur Creative Commons Lizenz beifügen und angeben, ob Änderungen vorgenommen wurden.

Die in diesem Kapitel enthaltenen Bilder und sonstiges Drittmaterial unterliegen ebenfalls der genannten Creative Commons Lizenz, sofern sich aus der Abbildungslegende nichts anderes ergibt. Sofern das betreffende Material nicht unter der genannten Creative Commons Lizenz steht und die betreffende Handlung nicht nach gesetzlichen Vorschriften erlaubt ist, ist für die oben aufgeführten Weiterverwendungen des Materials die Einwilligung des jeweiligen Rechteinhabers einzuholen.

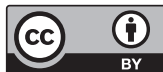

